Pacific

Journal of

Mathematics

2-BLOCKS WITH MINIMAL NONABELIAN DEFECT GROUPS III

\author{
BENJAMIN SAMBALE
}

Volume $280 \quad$ No. 2

February 2016 


\title{
2-BLOCKS WITH MINIMAL NONABELIAN DEFECT GROUPS III
}

\author{
BENJAMIN SAMBALE
}

\begin{abstract}
We prove that two 2-blocks of (possibly different) finite groups with a common minimal nonabelian defect group and the same fusion system are isotypic (and therefore perfectly isometric) in the sense of Broué. This continues former work by Cabanes and Picaronny (J. Fac. Sci. Univ. Tokyo Sect. IA Math. 39:1 (1992), 141-161), Sambale (J. Algebra 337 (2011), 261-284) and Eaton et al. (J. Group Theory 15:3 (2012), 311-321).
\end{abstract}

\section{Introduction}

Since its appearance in 1990, Broué's abelian defect conjecture gained much attention among representation theorists. On the level of characters it predicts the existence of a perfect isometry between a block with abelian defect group and its Brauer correspondent. These blocks have a common defect group and the same fusion system. Although Broué's conjecture is false for nonabelian defect groups (see [Cliff 2000]), one can still ask if perfect isometries or even isotypies exist. We affirmatively answer this question for $p=2$ and minimal nonabelian defect groups (see Theorem 9 below). These are the nonabelian defect groups such that any proper subgroup is abelian. Doing so, we verify the character-theoretic version of Rouquier's conjecture [2001, A.2] in this special case (see Corollary 10 below). At the same time we provide a new infinite family of defect groups supporting a blockwise $Z^{*}$-Theorem.

By Rédei's classification of minimal nonabelian $p$-groups, one has to consider three distinct families of defect groups. For two of these families the result already appeared in the literature (see [Cabanes and Picaronny 1992; Sambale 2011; Eaton et al. 2012]). Hence, it suffices to handle the remaining family which we will do in the next section. The proof of the main result is an application of [Horimoto and Watanabe 2012, Theorem 2]. The last section of the present paper also contains a related result for the nonabelian defect group of order 27 and exponent 9.

Our notation is fairly standard. We consider blocks $B$ of finite groups with respect to a $p$-modular system $(K, \mathcal{O}, F)$ where $\mathcal{O}$ is a complete discrete valuation

MSC2010: 20C15, 20C20.

Keywords: minimal nonabelian defect groups, perfect isometries, isotypies. 
ring with quotient field $K$ of characteristic 0 and field of fractions $F$ of characteristic $p$. As usual, we assume that $K$ is "large" enough and $F$ is algebraically closed. The number of irreducible ordinary characters (resp. Brauer characters) of $B$ is denoted by $k(B)$ (resp. $l(B)$ ). Moreover, $k_{i}(B)$ is the number of those irreducible characters of $B$ which have height $i \geq 0$. For other results on block invariants and fusion systems we often refer to [Sambale 2014]. Moreover, for the definition and construction of perfect isometries we follow [Broué and Puig 1980a; Cabanes and Picaronny 1992]. A cyclic group of order $n \in \mathbb{N}$ is denoted by $C_{n}$.

\section{A class of minimal nonabelian defect groups}

Let $B$ be a non-nilpotent 2-block of a finite group $G$ with defect group

$$
D=\left\langle x, y \mid x^{2^{r}}=y^{2}=[x, y]^{2}=[x, x, y]=[y, x, y]=1\right\rangle \cong C_{2}^{2} \rtimes C_{2^{r}}
$$

where $r \geq 2,[x, y]:=x y x^{-1} y^{-1}$ and $[x, x, y]:=[x,[x, y]]$.

We have already investigated some properties of $B$ in [Sambale 2011], and later gave simplified proofs in [Sambale 2014, Chapter 12]. For the convenience of the reader we restate some of these results.

Lemma 1 [Sambale 2014, Lemma 12.3]. Let $z:=[x, y]$. Then:

(i) $\Phi(D)=\mathrm{Z}(D)=\left\langle x^{2}, z\right\rangle \cong C_{2^{r-1}} \times C_{2}$.

(ii) $D^{\prime}=\langle z\rangle \cong C_{2}$.

(iii) $|\operatorname{Irr}(D)|=5 \cdot 2^{r-1}$.

Recall that a (saturated) fusion system $\mathcal{F}$ on a $p$-group $P$ determines the following subgroups:

$$
\begin{aligned}
\mathrm{Z}(\mathcal{F}) & :=\{x \in P: x \text { is fixed by every morphism in } \mathcal{F}\}, \\
\mathfrak{f o c}(\mathcal{F}) & :=\left\langle f(x) x^{-1}: x \in Q \leq P, f \in \operatorname{Aut}_{\mathcal{F}}(Q)\right\rangle, \\
\mathfrak{h} \mathfrak{y} \mathfrak{p}(\mathcal{F}) & :=\left\langle f(x) x^{-1}: x \in Q \leq P, f \in \mathrm{O}^{p}\left(\operatorname{Aut}_{\mathcal{F}}(Q)\right)\right\rangle .
\end{aligned}
$$

Lemma 2. The fusion system $\mathcal{F}$ of $B$ is the constrained fusion system of the finite group $A_{4} \rtimes C_{2^{r}}$ where $C_{2^{r}}$ acts as a transposition in $\operatorname{Aut}\left(A_{4}\right) \cong S_{4}$. In particular, $B$ has inertial index 1 and $Q:=\left\langle x^{2}, y, z\right\rangle \cong C_{2^{r-1}} \times C_{2}^{2}$ is the only $\mathcal{F}$-essential subgroup of $D$. Moreover, $\operatorname{Aut}_{\mathcal{F}}(Q) \cong S_{3}$. Without loss of generality, $\mathrm{Z}(\mathcal{F})=\left\langle x^{2}\right\rangle$ and $\mathfrak{h y p}(B)=\mathfrak{f o c}(B)=\mathfrak{f o c}(\mathcal{F})=\langle y, z\rangle$.

Proof. We have seen in [Sambale 2014, Proposition 12.7] that $\mathcal{F}$ is constrained and coincides with the fusion system of $A_{4} \rtimes C_{2^{r}}$. The construction of the semidirect product $A_{4} \rtimes C_{2^{r}}$ is slightly different in [Sambale 2014], but it is easy to see that both constructions give isomorphic groups. The remaining claims follow from the proof of [Sambale 2014, Proposition 12.7]. 
By a result of Watanabe [2014, Theorem 3 and Lemma 3], the hyperfocal subgroup of a 2-block is trivial or noncyclic. Hence, our situation with a Klein-four (hyper)focal subgroup represents the first nontrivial example in some sense. Recall that a $B$-subsection is a pair $\left(u, b_{u}\right)$ such that $u \in D$ and $b_{u}$ is a Brauer correspondent of $B$ in $\mathrm{C}_{G}(u)$.

Lemma 3. The set $\mathcal{R}:=\mathrm{Z}(D) \cup\left\{x^{i} y^{j}: i, j \in \mathbb{Z}\right.$, $i$ odd $\}$ is a set of representatives for the $\mathcal{F}$-conjugacy classes of $D$ with $|\mathcal{R}|=2^{r+1}$. For $u \in \mathcal{R}$ let $\left(u, b_{u}\right)$ be a $B$-subsection. Then $b_{u}$ has defect group $\mathrm{C}_{D}(u)$. Moreover, $l\left(b_{u}\right)=1$ whenever $u \in \mathcal{R} \backslash\left\langle x^{2}\right\rangle$.

Proof. By Lemma 2, it is easy to see that $\mathcal{R}$ is in fact a set of representatives for the $\mathcal{F}$-conjugacy classes of $D$. Observe that $\langle u\rangle$ is fully $\mathcal{F}$-normalized for all $u \in \mathcal{R}$. Hence, by [Sambale 2014, Lemma 1.34], $b_{u}$ has defect group $\mathrm{C}_{D}(u)$ and fusion system $\mathrm{C}_{\mathcal{F}}(\langle u\rangle)$. It is easy to see that $\mathrm{C}_{\mathcal{F}}(\langle u\rangle)$ is trivial unless $u \in \mathrm{Z}(\mathcal{F})=\left\langle x^{2}\right\rangle$. This shows $l\left(b_{u}\right)=1$ for $u \in \mathcal{R} \backslash\left\langle x^{2}\right\rangle$.

Theorem 4 [Sambale 2014, Theorem 12.4]. We have $k(B)=5 \cdot 2^{r-1}, k_{0}(B)=2^{r+1}$, $k_{1}(B)=2^{r-1}$ and $l(B)=2$.

Proof. By Lemma 2, we have $|D: \mathfrak{f o c}(B)|=2^{r}$. In particular, $2^{r} \mid k_{0}(B)$ by [Robinson 2008, Theorem 1]. Moreover, [Kessar et al. 2015, Theorem 1.1] implies $2^{r+1} \leq k_{0}(B)$. By Lemma 3 we have $l\left(b_{x}\right)=1$. Thus, we obtain $k_{0}(B)=2^{r+1}$ by a result of Robinson (see [Sambale 2014, Theorem 4.12]). In order to determine $l(B)$, we use induction on $r$. Let $u:=x^{2}$. Then $b_{u}$ dominates a block $\overline{b_{u}}$ of $\mathrm{C}_{G}(u) /\langle u\rangle$ with defect group $\bar{D}:=D /\langle u\rangle \cong D_{8}$ and fusion system $\overline{\mathcal{F}}:=\mathcal{F} /\langle u\rangle$. By [Linckelmann 2007, Theorem 6.3], $\left\langle x^{2}, y, z\right\rangle /\langle u\rangle \cong C_{2}^{2}$ is the only $\overline{\mathcal{F}}$-essential subgroup of $\bar{D}$. Therefore, a result of Brauer (see [Sambale 2014, Theorem 8.1]) shows that $l\left(b_{u}\right)=l\left(\overline{b_{u}}\right)=2$. By Lemma 3 and [Sambale 2014, Theorem 1.35] it follows that $k(B)>k_{0}(B)$. Since $|\mathrm{Z}(D): \mathrm{Z}(D) \cap \mathfrak{f o c}(B)|=2^{r-1}$, we have $2^{r-1} \mid k_{i}(B)$ for $i \geq 1$ by [Robinson 2008, Theorem 2]. Thus, by [Robinson 1991, Theorem 3.4] we obtain

$$
2^{r+2} \leq k_{0}(B)+4\left(k(B)-k_{0}(B)\right) \leq \sum_{i=0}^{\infty} k_{i}(B) 2^{2 i} \leq|D|=2^{r+2} .
$$

This gives $k_{1}(B)=2^{r-1}$ and $k(B)=k_{0}(B)+k_{1}(B)=5 \cdot 2^{r-1}$. In case $r=2$, [Sambale 2014, Theorem 1.35] implies

$$
l(B)=k(B)-\sum_{1 \neq u \in \mathcal{R}} l\left(b_{u}\right)=10-8=2 .
$$

Now let $r \geq 3$ and $1 \neq\langle u\rangle<\left\langle x^{2}\right\rangle$. Then $\overline{b_{u}}$ as above has the same type of defect group as $B$ except that $r$ is smaller. Hence, induction gives $l\left(b_{u}\right)=l\left(\overline{b_{u}}\right)=2$. Now the claim $l(B)=2$ follows again by [Sambale 2014, Theorem 1.35]. 
In the following results we denote the set of irreducible characters of $B$ of height $i$ by $\operatorname{Irr}_{i}(B)$.

Proposition 5 [Sambale 2014, Proposition 12.9]. The set $\operatorname{Irr}_{0}(B)$ contains four 2-rational characters and two families of 2-conjugate characters of size $2^{i}$ for every $i=1, \ldots, r-1$. The characters of height 1 split into two 2 -rational characters and one family of 2-conjugate characters of size $2^{i}$ for every $i=2, \ldots, r-2$.

Proposition 6. There are 2-rational characters $\chi_{i} \in \operatorname{Irr}(B)$ for $i=1,2,3$ such that

$$
\begin{aligned}
& \operatorname{Irr}_{0}(B)=\left\{\chi_{i} * \lambda: i=1,2, \lambda \in \operatorname{Irr}(D / \mathfrak{f o c}(B))\right\}, \\
& \operatorname{Irr}_{1}(B)=\left\{\chi_{3} * \lambda: \lambda \in \operatorname{Irr}(\mathrm{Z}(D) \mathfrak{f o c}(B) / \mathfrak{f o c}(B))\right\} .
\end{aligned}
$$

In particular, the characters of height 1 have the same degree and

$$
\left|\left\{\chi(1): \chi \in \operatorname{Irr}_{0}(B)\right\}\right| \leq 2 .
$$

Proof. We have already seen in the proof of Theorem 4 that the action of $D / \mathfrak{f o c}(B)$ on $\operatorname{Irr}_{0}(B)$ via the $*$-construction has two orbits, and the action of $\mathrm{Z}(D) \mathfrak{f o c}(B) / \mathfrak{f o c}(B)$ on $\operatorname{Irr}_{1}(B)$ is regular. By Proposition 5 we can choose 2-rational representatives for these orbits, having identified the sets $\operatorname{Irr}(D / \mathfrak{f o c}(B))$ and $\operatorname{Irr}(\mathrm{Z}(D) \mathfrak{f o c}(B) / \mathfrak{f o c}(B))$ with subsets of $\operatorname{Irr}(D)$ in an obvious manner.

In the situation of Proposition 6 it is conjectured that $\chi_{1}(1) \neq \chi_{2}(1)$ (see [Malle and Navarro 2011]).

Proposition 7 [Sambale 2014, Proposition 12.8]. The Cartan matrix of B is given by

$$
2^{r-1}\left(\begin{array}{ll}
3 & 1 \\
1 & 3
\end{array}\right)
$$

up to basic sets.

Observe that Proposition 7 also gives the Cartan matrix for the defect group $D_{8}$ and the corresponding fusion system (this would be the case $r=1$ ).

Now we are in a position to obtain the generalized decomposition matrix of $B$. This completes partial results in [Sambale 2011, Section 3.3].

Proposition 8. Let $\mathcal{R}$ and $\chi_{i}$ be as in Lemma 3 and Proposition 6 respectively. Then there are basic sets for $b_{u}(u \in \mathcal{R})$ and signs $\epsilon, \sigma \in\{ \pm 1\}$ such that the generalized decomposition numbers of $B$ have the following form:

\begin{tabular}{c|cccc}
$u$ & $x^{2 i}$ & $x^{2 i} z$ & $x^{2 i+1}$ & $x^{2 i+1} y$ \\
\hline$d_{\chi_{1} \varphi}^{u}$ & $(1,0)$ & 1 & 1 & 1 \\
$d_{\chi_{2} \varphi}^{u}$ & $(0, \epsilon)$ & $\epsilon$ & $\epsilon$ & $-\epsilon$ \\
$d_{\chi_{3} \varphi}^{u}$ & $(\sigma, \sigma)$ & $-2 \sigma$ & 0 & 0
\end{tabular}


Proof. Since the Galois group of $\mathbb{Q}\left(e^{2 \pi i / 2^{r}}\right)$ over $\mathbb{Q}$ acts on the columns of the generalized decomposition matrix (see Proposition 5), we only need to determine the numbers $d_{\chi_{i} \varphi}^{u}$ for $u \in\left\{x, x y, x^{2^{j}}, x^{2^{j}} z\right\}(i=1,2,3, j=1, \ldots, r)$. First let $u=x$. Then the orthogonality relations show that

$$
2^{r}\left|d_{\chi_{1} \varphi}^{x}\right|^{2}+2^{r}\left|d_{\chi_{2} \varphi}^{x}\right|^{2}+2^{r-1}\left|d_{\chi_{3} \varphi}^{x}\right|^{2}=2^{r+1} .
$$

Since $\chi_{1}$ and $\chi_{2}$ have height 0 , we have $d_{\chi_{1} \varphi}^{x} \neq 0 \neq d_{\chi_{2} \varphi}^{x}$ (see [Sambale 2014, Proposition 1.36]). It follows that $d_{\chi_{i} \varphi}^{x}= \pm 1$ for $i=1,2$ and $d_{\chi_{3} \varphi}^{x}=0$, because $\chi_{i}$ is 2-rational. By replacing $\varphi$ with $-\varphi$ if necessary (i.e., changing the basic set for $b_{x}$ ), we may assume that $d_{\chi_{1} \varphi}^{x}=1$. We set $d_{\chi_{2} \varphi}^{x}=: \epsilon_{0}$. Similarly, we obtain $d_{\chi_{1} \varphi}^{x y}=1$, $d_{\chi_{2} \varphi}^{x y}= \pm 1$ and $d_{\chi_{3} \varphi}^{x y}=0$. Now since the columns $d^{x}$ and $d^{x y}$ of the generalized decomposition matrix are orthogonal, we obtain $d_{x_{2} \varphi}^{x y}=-\epsilon_{0}$.

Now let $u:=x^{2^{j}}$ for some $j \in\{1, \ldots, r\}$. Let $\operatorname{IBr}\left(b_{u}\right)=\left\{\varphi_{1}, \varphi_{2}\right\}$ (see the proof of Theorem 4). Then by Proposition 7 we get

$$
\begin{aligned}
2^{r}\left|d_{\chi_{1} \varphi_{1}}^{u}\right|^{2}+2^{r}\left|d_{\chi_{2} \varphi_{1}}^{u}\right|^{2}+2^{r-1}\left|d_{\chi_{3} \varphi_{1}}^{u}\right|^{2} & =3 \cdot 2^{r-1}, \\
2^{r}\left|d_{\chi_{1} \varphi_{2}}^{u}\right|^{2}+2^{r}\left|d_{\chi_{2} \varphi_{2}}^{u}\right|^{2}+2^{r-1}\left|d_{\chi_{3} \varphi_{2}}^{u}\right|^{2} & =3 \cdot 2^{r-1}, \\
2^{r} d_{\chi_{1} \varphi_{1}}^{u} \overline{d_{\chi_{1} \varphi_{2}}^{u}}+2^{r} d_{\chi_{2} \varphi_{1}}^{u} \overline{d_{\chi_{2} \varphi_{2}}^{u}}+2^{r-1} d_{\chi_{3} \varphi_{1}}^{u} \overline{d_{\chi_{3} \varphi_{2}}^{u}} & =2^{r-1} .
\end{aligned}
$$

Obviously, $d_{\chi_{1} \varphi_{1}}^{u} d_{\chi_{2} \varphi_{1}}^{u}=0$ and we may assume that $\left(d_{\chi_{1} \varphi_{1}}^{u}, d_{\chi_{1} \varphi_{2}}^{u}\right)=(1,0)$ and $\left(d_{\chi_{2} \varphi_{1}}^{u}, d_{\chi_{2} \varphi_{2}}^{u}\right)=\left(0, \epsilon_{j}\right)$ for a sign $\epsilon_{j} \in\{ \pm 1\}$. Moreover, $d_{\chi_{3} \varphi_{1}}^{u}=d_{\chi_{3} \varphi_{2}}^{u}=: \sigma_{j} \in\{ \pm 1\}$. Now let $u:=x^{2^{j}} z$. Then we have

$$
2^{r}\left|d_{\chi_{1} \varphi}^{u}\right|^{2}+2^{r}\left|d_{\chi_{2} \varphi}^{u}\right|^{2}+2^{r-1}\left|d_{\chi_{3} \varphi}^{u}\right|^{2}=2^{r+2} .
$$

It is known that $2 \mid d_{\chi_{3} \varphi}^{u} \neq 0$, since $b_{u}$ is major (see [Sambale 2014, Proposition 1.36]). This gives $d_{\chi_{1} \varphi}^{u}=1, d_{\chi_{2} \varphi}^{u}= \pm 1$ and $d_{\chi_{3} \varphi}^{u}= \pm 2$. By the orthogonality to $d^{x^{2^{j}}}$ we obtain that $d_{\chi_{3} \varphi}^{u}=-2 \sigma_{j}$ and $d_{\chi_{2} \varphi}^{u}=\epsilon_{j}$.

It remains to show that the signs $\epsilon_{j}$ and $\sigma_{j}$ do not depend on $j$. For this we consider characters $\lambda, \psi \in \operatorname{Irr}(D)$ whose values are given as follows:

\begin{tabular}{c|rrrr} 
& $x^{2^{j}}$ & $x^{2^{j}} z$ & $x$ & $x y$ \\
\hline$\lambda$ & 1 & 1 & 1 & -1 \\
$\psi$ & 2 & -2 & 0 & 0
\end{tabular}

Observe that $\psi$ is the inflation of the irreducible character of $D /\left\langle x^{2}\right\rangle \cong D_{8}$ of degree 2. It is easy to see that $(\lambda+\psi)\left(x^{2 k} y\right)=-1=1-2=(\lambda+\psi)\left(x^{2 k} z\right)$ for every $k \in \mathbb{Z}$. It follows that $\lambda+\psi$ is $\mathcal{F}$-stable, i.e., $(\lambda+\psi)(u)=(\lambda+\psi)(v)$ whenever $u$ and $v$ are $\mathcal{F}$-conjugate. By [Broué and Puig 1980a], $\chi_{1} *(\lambda+\psi)$ is a generalized character of $B$. In particular, the scalar product $\left(\chi_{1} *(\lambda+\psi), \chi_{3}\right)_{G}$ is an integer. This number can be computed by using the so-called contribution numbers $m_{\chi_{1} \chi_{3}}^{u}:=d_{\chi_{1}}^{u} C_{u}^{-1} \bar{d}_{\chi_{3}}^{u} \mathrm{~T}$ where $C_{u}$ is the Cartan matrix of $b_{u}$ and $d_{\chi_{i}}^{u}$ is the 
row of the generalized decomposition matrix corresponding to $\left(u, b_{u}\right)$ and $\chi_{i}$. For $u=x^{2^{j}}$ we have

$$
C_{u}^{-1}=2^{-r-2}\left(\begin{array}{rr}
3 & -1 \\
-1 & 3
\end{array}\right)
$$

by Proposition 7. This gives $m_{\chi_{1} \chi_{3}}^{u}=2^{-r-1} \sigma_{j}$. Similarly, $m_{\chi_{1} \chi_{3}}^{u}=-2^{-r-1} \sigma_{j}$ for $u=x^{2^{j}} z$. Thus, we obtain

$$
\begin{aligned}
\left(\chi_{1} *(\lambda+\psi), \chi_{3}\right)_{G} & =\sum_{u \in \mathcal{R}}(\lambda+\psi)(u) m_{\chi_{1} \chi_{3}}^{u}=\sum_{u \in Z(D)}(\lambda+\psi)(u) m_{\chi_{1} \chi_{3}}^{u} \\
& =(3+1)\left(2^{-r-1} \sigma_{r}+2^{-r-1} \sum_{j=1}^{r-1} \sigma_{j} 2^{r-j-1}\right) \\
& =2^{-r+1} \sigma_{r}+\sum_{j=1}^{r-1} \sigma_{j} 2^{-j} .
\end{aligned}
$$

If $\sigma_{1}=\sigma_{j}$ for some $j \neq 1$, then it follows immediately that $\sigma_{1}=\cdots=\sigma_{r}$ (otherwise the scalar product above is not an integer). Now suppose that $-\sigma_{1}=\sigma_{2}=$ $\cdots=\sigma_{r}$. In this case we replace $\chi_{3}$ by the 2-rational character $\chi_{3} * \tau$ where $\tau \in \operatorname{Irr}(\mathrm{Z}(D) \mathfrak{f o c}(B) / \mathfrak{f o c}(B))$ such that $\tau\left(x^{2}\right)=-1$. This changes $\sigma_{1}$, but does not affect $\sigma_{j}$ for $j>1$.

A similar argument with the scalar product $\left(\chi_{2} *(\lambda+\psi), \chi_{3}\right)_{G}$ implies that $\epsilon_{1}=\cdots=\epsilon_{r}$. In case $\epsilon_{0}=-\epsilon_{1}$, we replace $\chi_{2}$ by $\chi_{2} * \tau$ where $\tau \in \operatorname{Irr}(D / \mathfrak{f o c}(B))$ such that $\tau(x)=-1$. Observe again that this changes $\epsilon_{0}$, but keeps $\epsilon_{j}$ for $j>0$. This completes the proof.

\section{The main result}

Theorem 9. Let $B$ and $\tilde{B}$ be 2-blocks of (possibly different) finite groups with a common minimal nonabelian defect group and the same fusion system. Then $B$ and $\tilde{B}$ are isotypic (and therefore perfectly isometric).

Proof. We may assume that $B$ is not nilpotent by [Broué and Puig 1980b]. Let $D$ be a defect group of $B$ and $\tilde{B}$. If $|D|=8$, then the claim follows from [Cabanes and Picaronny 1992]. Now suppose that $D$ is given as in (1). We will attach a tilde to everything associated with $\tilde{B}$. By Proposition 8 and [Horimoto and Watanabe 2012, Theorem 2] there is a perfect isometry $I: \operatorname{CF}(G, B) \rightarrow \operatorname{CF}(\tilde{G}, \tilde{B})$ where $\operatorname{CF}(G, B)$ denotes the space of class functions with basis $\operatorname{Irr}(B)$ over $K$. It remains to show that $I$ is also an isotypy. In order to do so, we follow [Cabanes and Picaronny 1992, Section V.2]. For each $u \in D$ let $\mathrm{CF}\left(\mathrm{C}_{G}(u)_{2^{\prime}}, b_{u}\right)$ be the space of class functions on $\mathrm{C}_{G}(u)$ which vanish on the $p$-singular classes and are spanned by $\operatorname{IBr}\left(b_{u}\right)$. The 
decomposition map $d_{G}^{u}: \mathrm{CF}(G, B) \rightarrow \mathrm{CF}\left(\mathrm{C}_{G}(u)_{2^{\prime}}, b_{u}\right)$ is defined by

$$
d_{G}^{u}(\chi)(s):=\chi\left(e_{b_{u}} u s\right)=\sum_{\varphi \in \operatorname{IBr}\left(b_{u}\right)} d_{\chi \varphi}^{u} \varphi(s)
$$

for $\chi \in \operatorname{Irr}(B)$ and $s \in \mathrm{C}_{G}(u)_{2^{\prime}}$ where $e_{b_{u}}$ is the block idempotent of $b_{u}$ over $\mathcal{O}$. Then $I$ determines isometries

$$
I^{u}: \mathrm{CF}\left(\mathrm{C}_{G}(u)_{2^{\prime}}, b_{u}\right) \rightarrow \mathrm{CF}\left(\mathrm{C}_{\tilde{G}}(u)_{2^{\prime}}, \tilde{b}_{u}\right)
$$

by the equation $d_{\tilde{G}}^{u} \circ I=I^{u} \circ d_{G}^{u}$. Note that $I^{1}$ is the restriction of $I$. We need to show that $I^{u}$ can be extended to a perfect isometry $\widehat{I}^{u}: \operatorname{CF}\left(\mathrm{C}_{G}(u), b_{u}\right) \rightarrow$ $\mathrm{CF}\left(\mathrm{C}_{\tilde{G}}(u), \tilde{b}_{u}\right)$. Suppose first that $b_{u}$ is nilpotent. Then by Proposition $8, d_{G}^{u}\left(\chi_{1}\right)=$ $\epsilon \varphi$ and $d_{\tilde{G}}^{u}\left(I\left(\chi_{1}\right)\right)=\tilde{\epsilon} \tilde{\varphi}$ where $\operatorname{IBr}\left(b_{u}\right)=\{\varphi\}$ and $\operatorname{IBr}\left(\tilde{b}_{u}\right)=\{\tilde{\varphi}\}$ for some signs $\epsilon, \tilde{\epsilon} \in\{ \pm 1\}$. It follows that $I^{u}(\varphi)=\epsilon \tilde{\epsilon} \tilde{\varphi}$. Let $\psi \in \operatorname{Irr}_{0}\left(b_{u}\right)$ and $\tilde{\psi} \in \operatorname{Irr}_{0}\left(\tilde{b}_{u}\right)$ be 2-rational characters. Then it is well known that $\varphi=d_{\mathrm{C}_{G}(u)}^{1}(\psi)$ and $\operatorname{Irr}\left(b_{u}\right)=$ $\{\psi * \lambda: \lambda \in \operatorname{Irr}(D)\}$ (see [Broué and Puig 1980b]). Therefore, we may define $\widehat{I}^{u}$ by $\widehat{I^{u}}(\psi * \lambda):=\epsilon \tilde{\epsilon} \tilde{\psi} * \lambda$ for $\lambda \in \operatorname{Irr}(D)$. Then $\widehat{I}^{u}$ is a perfect isometry and

$$
\widehat{I^{u}}(\varphi)=\widehat{I}^{u}\left(d_{\mathrm{C}_{G}(u)}^{1}(\psi)\right)=d_{\mathrm{C}_{\tilde{G}}(u)}^{1}\left(\widehat{I}^{u}(\psi)\right)=\epsilon \tilde{\epsilon} d_{\mathrm{C}_{\tilde{G}}(u)}^{1}(\tilde{\psi})=\epsilon \tilde{\epsilon} \tilde{\varphi}=I^{u}(\varphi) .
$$

Hence, $\widehat{I^{u}}$ extends $I^{u}$. Moreover, $\widehat{I}^{u}$ does not depend on the generator of $\langle u\rangle$, since the signs $\epsilon$ and $\tilde{\epsilon}$ were defined by means of 2-rational characters.

Assume next that $b_{u}$ is non-nilpotent. Then $u \in\left\langle x^{2}\right\rangle$ and $b_{u}$ has defect group $D$. By Proposition 8, we can choose basic sets $\varphi_{1}, \varphi_{2}$ (resp. $\tilde{\varphi}_{1}, \tilde{\varphi}_{2}$ ) for $b_{u}$ (resp. $\left.\tilde{b}_{u}\right)$ such that $\varphi_{i}=d_{G}^{u}\left(\chi_{i}\right)$ and $\tilde{\varphi}_{i}=d_{\tilde{G}}^{u}\left(I\left(\chi_{i}\right)\right)$ for $i=1,2$. Then $I^{u}\left(\varphi_{i}\right)=\tilde{\varphi}_{i}$ for $i=1$, 2. Since the Cartan matrix of $b_{u}$ with respect to the basic set $\varphi_{1}, \varphi_{2}$ is already fixed (and given by Proposition 7), we find 2-rational characters $\psi_{i} \in \operatorname{Irr}_{0}\left(b_{u}\right)$ such that $d_{\mathrm{C}_{G}(u)}^{1}\left(\psi_{i}\right)=\epsilon_{i} \varphi_{i}$ with $\epsilon_{i} \in\{ \pm 1\}$ for $i=1,2$ (see the proof of Proposition 8). Similarly, one has $\tilde{\psi}_{i} \in \operatorname{Irr}_{0}\left(\tilde{b}_{u}\right)$ such that $d_{\mathrm{C}_{\tilde{G}}(u)}^{1}\left(\tilde{\psi}_{i}\right)=\tilde{\epsilon}_{i} \tilde{\varphi}_{i}$. Then, by what we have already shown, there exists a perfect isometry

$$
\widehat{I}^{u}: \mathrm{CF}\left(\mathrm{C}_{G}(u), b_{u}\right) \rightarrow \mathrm{CF}\left(\mathrm{C}_{\tilde{G}}(u), \tilde{b}_{u}\right)
$$

sending $\psi_{i}$ to $\epsilon_{i} \tilde{\epsilon}_{i} \tilde{\psi}_{i}$ for $i=1,2$. We have

$$
\widehat{I^{u}}\left(\varphi_{i}\right)=\epsilon_{i} \widehat{I}^{u}\left(d_{\mathrm{C}_{G}(u)}^{1}\left(\psi_{i}\right)\right)=\epsilon_{i} d_{\mathrm{C}_{\tilde{G}}(u)}^{1}\left(\widehat{I}^{u}\left(\psi_{i}\right)\right)=\tilde{\epsilon}_{i} d_{\mathrm{C}_{\tilde{G}}(u)}^{1}\left(\tilde{\psi}_{i}\right)=\tilde{\varphi}_{i}=I^{u}\left(\varphi_{i}\right)
$$

for $i=1,2$. This shows that $\widehat{I}^{u}$ extends $I^{u}$. Moreover, it is easy to see that $\widehat{I}^{u}$ does not depend on the generator of $\langle u\rangle$.

Altogether we have proved the theorem if $D$ is given as in (1). By [Sambale 2014, Theorem 12.4] it remains to handle the case

$$
D \cong\left\langle x, y \mid x^{2^{r}}=y^{2^{r}}=[x, y]^{2}=[x, x, y]=[y, x, y]=1\right\rangle
$$


where $r \geq 2$. Here $B$ and $\tilde{B}$ are Morita equivalent and therefore perfectly isometric. However, a Morita equivalence does not automatically provide an isotypy. Nevertheless, in this special case the Morita equivalence is a composition of various "natural" equivalences (namely Fong reductions, Külshammer-Puig reduction and Külshammer's reduction for blocks with normal defect groups, see [Eaton et al. 2012, proof of Theorem 1]). In particular, the generalized decomposition matrices of $B$ and $\tilde{B}$ coincide up to signs (see [Watanabe 1985]). Now we can use the same methods as above in order to construct an isotypy. In fact, for every $B$-subsection $\left(u, b_{u}\right)$ one has that $b_{u}$ is nilpotent or $u=[x, y]$ and $b_{u}$ is Morita equivalent to $B$ (see the proof of [Sambale 2011, Proposition 4.3]). We omit the details.

Corollary 10. Let $B$ be a 2-block of a finite group $G$ with minimal nonabelian defect group $D ¥ D_{8}$. Then $B$ is isotypic to a Brauer correspondent in $\mathrm{N}_{G}(\mathfrak{h y p}(B))$.

Proof. Let $b_{D}$ be a Brauer correspondent of $B$ in $D \mathrm{C}_{G}(D)$. Since $D \mathrm{C}_{G}(D) \subseteq$ $\mathrm{N}_{G}(\mathfrak{h y p}(B))$, the Brauer correspondent $b:=b_{D}^{\mathrm{N}_{G}(\mathfrak{h} \mathfrak{p} \mathfrak{p}(B))}$ of $B$ has defect group $D$. By Theorem 9, it suffices to show that $B$ and $b$ have the same fusion system. Observe that $\mathrm{N}_{G}\left(D, b_{D}\right) \subseteq \mathrm{N}_{G}(\mathfrak{h y p}(B))$. In particular, $B$ and $b$ have the same inertial quotient. If there is only the trivial fusion system on $D$, then we are done (this applies if $D$ is metacyclic of order at least 16). In case $D \cong Q_{8}, B$ is a controlled block (see, e.g., [Cabanes and Picaronny 1992]). Since $B$ and $b$ have the same inertial quotient, it follows that these blocks also have the same fusion system. It remains to consider the two other families of defect groups (see [Sambale 2014, Theorem 12.4]). For one of these families the fusion system is again controlled (see [Sambale 2014, Proposition 12.7]). Finally, if $D$ is given as in (1), then the fusion system is constrained and the automorphisms of the essential subgroup (if it exists) also act on $\mathfrak{h y p}(B)$. Hence, $B$ is nilpotent if and only if $b$ is nilpotent. Again the claim follows from Theorem 9 .

We remark that Corollary 10 would be false in case $D \cong D_{8}$. The principal 2-block of $\operatorname{GL}(3,2)$ gives a counterexample. If $B$ is a block of a finite group $G$ with defect group as given in (1), then $B$ is also isotypic to a Brauer correspondent in $\mathrm{C}_{G}(u)$ where $u \in \mathrm{Z}(\mathcal{F})$. This resembles Glauberman's $\mathrm{Z}^{*}$-theorem.

In the situation of Theorem 9 (or Corollary 10) it is desirable to extend the isotypies to Morita equivalences (as we did in [Eaton et al. 2012]). This is not always possible if $|D|=8$, since for example the principal 2-blocks of the symmetric groups $S_{4}$ and $S_{5}$ are not Morita equivalent. Nevertheless, the possible Morita equivalence classes in case $|D|=8$ are known by Erdmann's classification of tame algebra [Erdmann 1990] (at least over $F$, see [Holm 2001]). In view of [Eaton et al. 2012] one may still ask if two non-nilpotent 2-blocks with isomorphic defect groups as in Section 2 are Morita equivalent. We will see that the answer is again negative. 
Consider the groups $G_{1}:=A_{4} \rtimes C_{2^{r}}$ and $G_{2}:=A_{5} \rtimes C_{2^{r}}$ constructed similarly as in Lemma 2. Then $G_{1} / \mathrm{Z}\left(G_{1}\right) \cong S_{4}$ and $G_{2} / \mathrm{Z}\left(G_{2}\right) \cong S_{5}$. Let $B_{i}$ be the principal 2-block of $G_{i}$, and let $\overline{B_{i}}$ be the principal 2-block of $G_{i} / \mathrm{Z}\left(G_{i}\right)$ for $i=1,2$. Then the Cartan matrix of $B_{i}$ is just the Cartan matrix of $\overline{B_{i}}$ multiplied by $\left|\mathrm{Z}\left(G_{i}\right)\right|=2^{r-1}$. It is known that the Cartan matrices of $\overline{B_{1}}$ and $\overline{B_{2}}$ do not coincide (regardless of the labeling of the simple modules). Therefore, $B_{1}$ and $B_{2}$ are not Morita equivalent.

Nevertheless, the structure of a finite group $G$ with a minimal nonabelian Sylow 2 -subgroup $P$ as given in (1) is fairly restricted. More precisely, Glauberman's $\mathrm{Z}^{*}$-theorem implies $x^{2} \in \mathrm{Z}^{*}(G)$, and the structure of $G / \mathrm{Z}^{*}(G)$ follows from the Gorenstein-Walter theorem [1965]. In particular, $G$ has at most one nonabelian composition factor by Feit-Thompson.

We use the opportunity to present a related result for $p=3$ which extends [Sambale 2014, Theorem 8.15].

Theorem 11. Let $B$ and $\tilde{B}$ be non-nilpotent blocks of (possibly different) finite groups both with defect group $C_{9} \rtimes C_{3}$. Then $B$ and $\tilde{B}$ are isotypic.

Proof. As in the proof of Theorem 9, we will make use of [Horimoto and Watanabe 2012, Theorem 2]. Let

$$
D:=\left\langle x, y \mid x^{9}=y^{3}=1, y x y^{-1}=x^{4}\right\rangle
$$

be a defect group of $B$, and let $\mathcal{F}$ be the fusion system of $B$. By [Stancu 2006], $B$ is controlled with inertial index 2, and we may assume that $x$ and $x^{-1}$ are $\mathcal{F}$-conjugate (see the proof of [Sambale 2014, Theorem 8.8]). Then $\mathcal{R}:=\left\{1, x, x^{3}, y, y^{2}, x y, x y^{2}\right\}$ is a set of representatives for the $\mathcal{F}$-conjugacy classes of $D$ (see the proof of [Sambale 2014, Theorem 8.15]). It suffices to show that the generalized decomposition numbers of $B$ are essentially unique (up to basic sets and signs and permutations of rows). Since the Galois group of $\mathbb{Q}\left(e^{2 \pi i / 9}\right)$ over $\mathbb{Q}$ acts on the columns of the generalized decomposition matrix, we only need to determine the numbers $d_{\chi \varphi}^{u}$ for $u \in\left\{x, x^{3}, y, x y\right\}$. By [Sambale 2014, Theorem 8.15] there are four 3-rational characters $\chi_{i} \in \operatorname{Irr}(B)(i=1, \ldots, 4)$ such that $\chi_{1}, \chi_{2}$ and $\chi_{3}$ have height 0 and $\chi_{4}$ has height 1 . Since $\mathfrak{f o c}(B)=\langle x\rangle$, we see that

$$
\operatorname{Irr}(B)=\left\{\chi_{i} * \lambda: i=1,2,3, \lambda \in \operatorname{Irr}(D / \mathfrak{f o c}(B))\right\} \cup\left\{\chi_{4}\right\} .
$$

Let $u:=x^{3}$. Then $\operatorname{IBr}\left(b_{u}\right)=\{\varphi\}$ and $d_{\chi_{i} \varphi}^{u}$ are nonzero (rational) integers. Moreover, $d_{\chi_{4} \varphi}^{u} \equiv 0(\bmod 3)$. After permuting $\chi_{1}, \chi_{2}$ and $\chi_{3}$ and changing the basic set for $b_{u}$ if necessary, we may assume that $d_{\chi_{1} \varphi}^{u}=2, d_{\chi_{2} \varphi}^{u}=: \epsilon_{1} \in\{ \pm 1\}, d_{\chi_{3} \varphi}^{u}=: \epsilon_{2} \in\{ \pm 1\}$ and $d_{\chi_{4} \varphi}^{u}=3 \epsilon_{3} \in\{ \pm 3\}$. Now let $u:=x$. Then $d_{\chi_{i} \varphi}^{u}= \pm 1$ for $i=1,2,3$ and $d_{\chi_{4} \varphi}^{u}=0$. We may choose a basic set for $b_{u}$ such that $d_{\chi_{1} \varphi}^{u}=1$. Then by the orthogonality relations, $d_{\chi_{2} \varphi}^{u}=-\epsilon_{1}$ and $d_{\chi_{3} \varphi}^{u}=-\epsilon_{2}$. Next let $u:=y$. Then $b_{u}$ dominates a block of $\mathrm{C}_{G}(u) /\langle u\rangle$ with cyclic defect group $\mathrm{C}_{D}(u) /\langle u\rangle \cong C_{3}$ and inertial index 2 . This 
yields $\operatorname{IBr}\left(b_{u}\right)=\left\{\varphi_{1}, \varphi_{2}\right\}$ and the Cartan matrix of $b_{u}$ is given by

$$
3\left(\begin{array}{ll}
2 & 1 \\
1 & 2
\end{array}\right)
$$

(not only up to basic sets, but this is not important here). We can choose a basic set such that $\left(d_{\chi_{1} \varphi_{1}}^{u}, d_{\chi_{1} \varphi_{2}}^{u}\right)=(1,1),\left(d_{\chi_{2} \varphi_{1}}^{u}, d_{\chi_{2} \varphi_{2}}^{u}\right)=\left(\sigma_{1}, 0\right),\left(d_{\chi_{3} \varphi_{1}}^{u}, d_{\chi_{3} \varphi_{2}}^{u}\right)=\left(0, \sigma_{2}\right)$ and $\left(d_{\chi_{4} \varphi_{1}}^{u}, d_{\chi_{4} \varphi_{2}}^{u}\right)=(0,0)$ for some signs $\sigma_{1}, \sigma_{2} \in\{ \pm 1\}$. Finally for $u:=x y$ we obtain $d_{\chi_{1} \varphi}^{u}=1, d_{\chi_{i} \varphi}^{u}=-\sigma_{i-1}$ for $i=2,3$ and $d_{\chi_{4} \varphi}^{u}=0$ after changing the basic set if necessary. The following table summarizes the results:

\begin{tabular}{c|rrrr}
$u$ & $x^{3}$ & $x$ & $y$ & $x y$ \\
\hline$d_{\chi_{1} \varphi}^{u}$ & 2 & 1 & $(1,1)$ & 1 \\
$d_{\chi_{2} \varphi}^{u}$ & $\epsilon_{1}$ & $-\epsilon_{1}$ & $\left(\sigma_{1}, 0\right)$ & $-\sigma_{1}$ \\
$d_{\chi_{3} \varphi}^{u}$ & $\epsilon_{2}$ & $-\epsilon_{2}$ & $\left(0, \sigma_{2}\right)$ & $-\sigma_{2}$ \\
$d_{\chi_{4} \varphi}^{u}$ & $3 \epsilon_{3}$ & 0 & $(0,0)$ & 0
\end{tabular}

It suffices to show that $\epsilon_{i}=\sigma_{i}$ for $i=1,2$ (observe that we do not need the ordinary decomposition numbers in order to apply [Horimoto and Watanabe 2012, Theorem 2]). For this, let $\lambda \in \operatorname{Irr}\left(D /\left\langle x^{3}\right\rangle\right)$ such that $\lambda(x)=e^{2 \pi i / 3}$ and $\lambda(y)=1$. Then the generalized character $\psi:=\lambda+\bar{\lambda}-2 \cdot 1_{D}$ of $D$ is constant on $\langle x\rangle \backslash\left\langle x^{3}\right\rangle$ and thus $\mathcal{F}$-stable. By [Broué and Puig 1980a], $\chi_{1} * \psi$ is a generalized character of $B$ and $\left(\chi_{1} * \psi, \chi_{2}\right)_{G} \in \mathbb{Z}$. As in the proof of Theorem 9, we compute

$$
\begin{aligned}
\left(\chi_{1} * \psi, \chi_{2}\right)_{G} & =\sum_{u \in \mathcal{R}} \psi(u) m_{\chi_{1} \chi_{2}}^{u}=\psi(x) m_{\chi_{1} \chi_{2}}^{x}+\psi(x y) m_{\chi_{1} \chi_{2}}^{x y}+\psi\left(x y^{2}\right) m_{\chi_{1} \chi_{2}}^{x y^{2}} \\
& =\frac{1}{3} \epsilon_{1}+\frac{2}{3} \sigma_{1} .
\end{aligned}
$$

This shows $\epsilon_{1}=\sigma_{1}$. Similarly, one gets $\epsilon_{2}=\sigma_{2}$ by computing $\left(\chi_{1} * \psi, \chi_{3}\right)_{G}$. Hence, [Horimoto and Watanabe 2012, Theorem 2] gives a perfect isometry $I: \mathrm{CF}(G, B) \rightarrow$ $\mathrm{CF}(\tilde{G}, \tilde{B})$. In order to show that $I$ is also an isotypy, we make use of the notation introduced in the proof of Theorem 9. Let $u \in D$ such that $b_{u}$ is nilpotent. Then by the table above, we have $\operatorname{IBr}\left(b_{u}\right)=\left\{ \pm d_{G}^{u}\left(\chi_{2}\right)\right\}$. Thus, one can extend $I^{u}$ just as in Theorem 9. Now suppose that $b_{u}$ is non-nilpotent and thus $u=y$ (up to inversion). We choose a basic set $\varphi_{1}, \varphi_{2}$ for $b_{u}$ as above such that $d_{G}^{u}\left(\chi_{i}\right)=\varphi_{i-1}$ for $i=2,3$. Now we have to determine the ordinary decomposition numbers of $b_{u}$ with respect to $\varphi_{1}, \varphi_{2}$. The defect group of $b_{u}$ is $\mathrm{C}_{D}(y)=\left\langle x^{3}, y\right\rangle \cong C_{3} \times C_{3}$ and $\mathfrak{f o c}\left(b_{u}\right)=\left\langle x^{3}\right\rangle$. By [Kiyota 1984], $k\left(b_{u}\right)=9$. Therefore, there are 3-rational characters $\psi_{i} \in \operatorname{Irr}\left(b_{u}\right)$ such that

$$
\operatorname{Irr}\left(b_{u}\right)=\left\{\psi_{i} * \lambda: i=1,2,3, \lambda \in \operatorname{Irr}\left(\left\langle x^{3}, y\right\rangle /\left\langle x^{3}\right\rangle\right)\right\}
$$

By the Cartan matrix of $b_{u}$ given above (with respect to $\varphi_{1}, \varphi_{2}$ ), it follows immediately that $d_{\mathrm{C}_{G}(u)}^{1}\left(\psi_{i}\right)=\epsilon_{i} \varphi_{i}$ with $\epsilon_{i} \in\{ \pm 1\}$ for $i=1,2$ after a suitable permutation of 
$\psi_{1}, \psi_{2}, \psi_{3}$. Similarly, $d_{\mathrm{C}_{\tilde{G}}(u)}^{1}\left(\tilde{\psi}_{i}\right)=\tilde{\epsilon}_{i} \tilde{\varphi}_{i}$. By a result of Usami [1988], there is a perfect isometry $\mathrm{CF}\left(\mathrm{C}_{G}(u), b_{u}\right) \rightarrow \mathrm{CF}\left(\mathrm{C}_{\tilde{G}}(u), \tilde{b}_{u}\right)$. However, we need the additional information that $\psi_{i}$ is mapped to $\pm \tilde{\psi}_{i}$. In order to show this, we use [Horimoto and Watanabe 2012, Theorem 2] again. Observe that $d_{\mathrm{C}_{G}(u)}^{u}\left(\psi_{i}\right)=\zeta_{i} d_{\mathrm{C}_{G}(u)}^{1}\left(\psi_{i}\right)=\zeta_{i} \epsilon_{i} \varphi_{i}$ for a cube root of unity $\zeta_{i}$. But since $d_{\psi_{i} \varphi_{i}}^{u}$ is rational, we have $\zeta_{i}=1$. Now an elementary application of the orthogonality relations shows that the generalized decomposition matrix of $b_{u}$ (in $\mathrm{C}_{G}(u)$ ) is determined by

\begin{tabular}{c|ccrr}
$v$ & 1 & $y$ & $x^{3}$ & $x^{3} y$ \\
\hline$d_{\psi_{1} \varphi}^{v}$ & $\left(\epsilon_{1}, 0\right)$ & $\left(\epsilon_{1}, 0\right)$ & $\epsilon_{1}$ & $\epsilon_{1}$ \\
$d_{\psi_{2} \varphi}$ & $\left(0, \epsilon_{2}\right)$ & $\left(0, \epsilon_{2}\right)$ & $\epsilon_{2}$ & $\epsilon_{2}$ \\
$d_{\psi_{3} \varphi}^{v}$ & $\left(\epsilon_{3}, \epsilon_{3}\right)$ & $\left(\epsilon_{3}, \epsilon_{3}\right)$ & $-\epsilon_{3}$ & $-\epsilon_{3}$
\end{tabular}

It follows that there is a perfect isometry $\widehat{I}^{u}: \mathrm{CF}\left(\mathrm{C}_{G}(u), b_{u}\right) \rightarrow \mathrm{CF}\left(\mathrm{C}_{\tilde{G}}(u), \tilde{b}_{u}\right)$ such that $\widehat{I^{u}}\left(\psi_{i}\right)=\epsilon_{i} \tilde{\epsilon}_{i} \tilde{\psi}_{i}$ for $i=1,2$. Therefore $\widehat{I}^{u}$ extends $I^{u}$. As in the proof of Theorem 9, it is also clear that $\widehat{I}^{u}$ is independent of the choice of the generator of $\langle u\rangle$. This finishes the proof.

The proof method of Theorem 11 also works for other defect groups. In fact, Watanabe [2015] showed independently (using more complicated methods) that two $p$-blocks $(p>2)$ with a common metacyclic, minimal nonabelian defect group and the same fusion system are perfectly isometric. Again, this gives evidence for the character-theoretic version of Rouquier's conjecture (see [Watanabe 2014, Theorem 2]). As another remark, Holloway, Koshitani and Kunugi [2010, Example 4.3] constructed a perfect isometry between the principal 3-block of $G:=\operatorname{Aut}(\operatorname{SL}(2,8)) \cong{ }^{2} G_{2}(3)$ and its Brauer correspondent. Since $G$ has a Sylow 3 -subgroup isomorphic to $C_{9} \rtimes C_{3}$, this is a special case of Theorem 11. Note that in the introduction of [Ruengrot 2011] it is erroneously stated that these blocks are not perfectly isometric.

\section{Acknowledgment}

This work is supported by the German Research Foundation and the Daimler and Benz Foundation. The author thanks Atumi Watanabe for providing a copy of [Watanabe 2015]. Moreover, the author thanks Burkhard Külshammer for answering some questions.

\section{References}

[Broué and Puig 1980a] M. Broué and L. Puig, "Characters and local structure in $G$-algebras", $J$. Algebra 63:2 (1980), 306-317. MR 81j:20021 Zbl 0428.20005

[Broué and Puig 1980b] M. Broué and L. Puig, "A Frobenius theorem for blocks", Invent. Math. 56:2 (1980), 117-128. MR 81d:20011 Zbl 0425.20008 
[Cabanes and Picaronny 1992] M. Cabanes and C. Picaronny, "Types of blocks with dihedral or quaternion defect groups", J. Fac. Sci. Univ. Tokyo Sect. IA Math. 39:1 (1992), 141-161. MR 93f:20014 Zbl 0781.20006

[Cliff 2000] G. Cliff, “On centers of 2-blocks of Suzuki groups”, J. Algebra 226:1 (2000), 74-90. MR 2001d:20013 Zbl 0953.20003

[Eaton et al. 2012] C. W. Eaton, B. Külshammer, and B. Sambale, "2-blocks with minimal nonabelian defect groups, II”, J. Group Theory 15:3 (2012), 311-321. MR 2920888 Zbl 1253.20008

[Erdmann 1990] K. Erdmann, Blocks of tame representation type and related algebras, Lecture Notes in Mathematics 1428, Springer, Berlin, 1990. MR 91c:20016 Zbl 0696.20001

[Gorenstein and Walter 1965] D. Gorenstein and J. H. Walter, "The characterization of finite groups with dihedral Sylow 2-subgroups, I", J. Algebra 2 (1965), 85-151. MR 31 \#1297a Zbl 0192.11902

[Holloway et al. 2010] M. Holloway, S. Koshitani, and N. Kunugi, "Blocks with nonabelian defect groups which have cyclic subgroups of index p", Arch. Math. (Basel) 94:2 (2010), 101-116. MR 2011c:20013 Zbl 1195.20010

[Holm 2001] T. Holm, "Notes on Donovan's Conjecture for blocks of tame representation type", 2001, http://www2.iazd.uni-hannover.de/ tholm/ARTIKEL/donovan.ps. Unpublished notes.

[Horimoto and Watanabe 2012] H. Horimoto and A. Watanabe, "On a perfect isometry between principal p-blocks of finite groups with cyclic p-hyperfocal subgroups", preprint, 2012, http:// hdl.handle.net/2433/194497. In Japanese.

[Kessar et al. 2015] R. Kessar, M. Linckelmann, and G. Navarro, "A characterisation of nilpotent blocks", Proc. Amer. Math. Soc. (online publication June 2015).

[Kiyota 1984] M. Kiyota, "On 3-blocks with an elementary abelian defect group of order 9", J. Fac. Sci. Univ. Tokyo Sect. IA Math. 31:1 (1984), 33-58. MR 85k:20036 Zbl 0546.20013

[Linckelmann 2007] M. Linckelmann, "Introduction to fusion systems", pp. 79-113 in Group representation theory, edited by M. Geck et al., EPFL Press, Lausanne, 2007. MR 2008f:20021 Zbl 1161.20007

[Malle and Navarro 2011] G. Malle and G. Navarro, "Blocks with equal height zero degrees", Trans. Amer. Math. Soc. 363:12 (2011), 6647-6669. MR 2012g:20016 Zbl 1277.20013

[Robinson 1991] G. R. Robinson, "On the number of characters in a block", J. Algebra 138:2 (1991), 515-521. MR 92h:20022a Zbl 0727.20010

[Robinson 2008] G. R. Robinson, "On the focal defect group of a block, characters of height zero, and lower defect group multiplicities", J. Algebra 320:6 (2008), 2624-2628. MR 2009f:20010 Zbl 1153.20006

[Rouquier 2001] R. Rouquier, "Block theory via stable and Rickard equivalences", pp. 101-146 in Modular representation theory of finite groups (Charlottesville, VA, 1998), edited by M. J. Collins et al., de Gruyter, Berlin, 2001. MR 2003g:20018 Zbl 0998.20006

[Ruengrot 2011] P. Ruengrot, Perfect isometry groups for blocks of finite groups, Ph.D. thesis, University of Manchester, 2011, https://www.escholar.manchester.ac.uk/uk-ac-man-scw:142297.

[Sambale 2011] B. Sambale, "2-blocks with minimal nonabelian defect groups", J. Algebra 337 (2011), 261-284. MR 2012d:20019 Zbl 1247.20010

[Sambale 2014] B. Sambale, Blocks of finite groups and their invariants, Lecture Notes in Mathematics 2127, Springer, Berlin, 2014. MR 3289382 Zbl 1315.20009

[Stancu 2006] R. Stancu, "Control of fusion in fusion systems", J. Algebra Appl. 5:6 (2006), 817-837. MR 2007j:20025 Zbl 1118.20020 
[Usami 1988] Y. Usami, "On p-blocks with abelian defect groups and inertial index 2 or 3, I", J. Algebra 119:1 (1988), 123-146. MR 89i:20024 Zbl 0659.20008

[Watanabe 1985] A. Watanabe, "On generalized decomposition numbers and Fong's reductions", Osaka J. Math. 22:2 (1985), 393-400. MR 86i:20018 Zbl 0575.20011

[Watanabe 2014] A. Watanabe, "The number of irreducible Brauer characters in a $p$-block of a finite group with cyclic hyperfocal subgroup”, J. Algebra 416 (2014), 167-183. MR 3232798 Zbl 06339539

[Watanabe 2015] A. Watanabe, "On blocks of finite groups with metacyclic, minimal non-abelian defect groups", preprint, 2015.

Received April 26, 2015. Revised June 6, 2015.

BENJAMIN SAMBALE

FACHBEREICH MATHEMATIK

TU KAISERSLAUTERN

67653 KAISERSLAUTERN

GERMANY

sambale@mathematik.uni-kl.de 


\title{
PACIFIC JOURNAL OF MATHEMATICS
}

\author{
msp.org/pjm
}

Founded in 1951 by E. F. Beckenbach (1906-1982) and F. Wolf (1904-1989)

\section{EDITORS}

Don Blasius (Managing Editor)

Department of Mathematics

University of California

Los Angeles, CA 90095-1555

blasius@math.ucla.edu

\author{
Paul Balmer \\ Department of Mathematics \\ University of California \\ Los Angeles, CA 90095-1555 \\ balmer@math.ucla.edu \\ Robert Finn \\ Department of Mathematics \\ Stanford University \\ Stanford, CA 94305-2125 \\ finn@math.stanford.edu \\ Sorin Popa \\ Department of Mathematics \\ University of California \\ Los Angeles, CA 90095-1555 \\ popa@math.ucla.edu
}

\author{
Vyjayanthi Chari \\ Department of Mathematics \\ University of California \\ Riverside, CA 92521-0135 \\ chari@math.ucr.edu \\ Kefeng Liu \\ Department of Mathematics \\ University of California \\ Los Angeles, CA 90095-1555 \\ liu@math.ucla.edu \\ Jie Qing \\ Department of Mathematics \\ University of California \\ Santa Cruz, CA 95064 \\ qing@ cats.ucsc.edu
}

\section{PRODUCTION}

Silvio Levy, Scientific Editor, production@msp.org

\section{SUPPORTING INSTITUTIONS}

ACADEMIA SINICA, TAIPEI

CALIFORNIA INST. OF TECHNOLOGY

INST. DE MATEMÁTICA PURA E APLICADA

KEIO UNIVERSITY

MATH. SCIENCES RESEARCH INSTITUTE

NEW MEXICO STATE UNIV.

OREGON STATE UNIV.

\author{
STANFORD UNIVERSITY \\ UNIV. OF BRITISH COLUMBIA \\ UNIV. OF CALIFORNIA, BERKELEY \\ UNIV. OF CALIFORNIA, DAVIS \\ UNIV. OF CALIFORNIA, LOS ANGELES \\ UNIV. OF CALIFORNIA, RIVERSIDE \\ UNIV. OF CALIFORNIA, SAN DIEGO \\ UNIV. OF CALIF., SANTA BARBARA
}

\author{
Daryl Cooper \\ Department of Mathematics \\ University of California \\ Santa Barbara, CA 93106-3080 \\ cooper@math.ucsb.edu \\ Jiang-Hua Lu \\ Department of Mathematics \\ The University of Hong Kong \\ Pokfulam Rd., Hong Kong \\ jhlu@maths.hku.hk \\ Paul Yang \\ Department of Mathematics \\ Princeton University \\ Princeton NJ 08544-1000 \\ yang@math.princeton.edu
}

These supporting institutions contribute to the cost of publication of this Journal, but they are not owners or publishers and have no responsibility for its contents or policies.

See inside back cover or msp.org/pjm for submission instructions.

The subscription price for 2016 is US $\$ 440 /$ year for the electronic version, and $\$ 600 /$ year for print and electronic.

Subscriptions, requests for back issues and changes of subscribers address should be sent to Pacific Journal of Mathematics, P.O. Box 4163, Berkeley, CA 94704-0163, U.S.A. The Pacific Journal of Mathematics is indexed by Mathematical Reviews, Zentralblatt MATH, PASCAL CNRS Index, Referativnyi Zhurnal, Current Mathematical Publications and Web of Knowledge (Science Citation Index).

The Pacific Journal of Mathematics (ISSN 0030-8730) at the University of California, c/o Department of Mathematics, 798 Evans Hall \#3840, Berkeley, CA 94720-3840, is published twelve times a year. Periodical rate postage paid at Berkeley, CA 94704, and additional mailing offices. POSTMASTER: send address changes to Pacific Journal of Mathematics, P.O. Box 4163, Berkeley, CA 94704-0163.

PJM peer review and production are managed by EditFLOW ${ }^{\circledR}$ from Mathematical Sciences Publishers.

\section{PUBLISHED BY}

\section{mathematical sciences publishers \\ nonprofit scientific publishing}

http://msp.org/

(C) 2016 Mathematical Sciences Publishers 


\section{PACIFIC JOURNAL OF MATHEMATICS}

Volume $280 \quad$ No. $2 \quad$ February 2016

Topological Molino's theory

JESÚS A. ÁlVAREz LóPEZ and MANUEl F. MoreIRA GALICIA

Equivariant principal bundles and logarithmic connections on toric varieties

INDRANIL BISWAS, ARIJIT DEY and MAINAK PODDAR

On a spectral theorem in paraorthogonality theory

Kenier CASTILlo, RuYmán CRUZ-BARRoso and Francisco

PERDOMO-PÍO

Sigma theory and twisted conjugacy, II: Houghton groups and pure

symmetric automorphism groups

DACIBERG L. GONÇALVES and PARAMESWARAN SANKARAN

The second CR Yamabe invariant

PAK TUNG Ho

No hyperbolic pants for the 4-body problem with strong potential

CONNOR JACKMAN and RICHARD MONTGOMERY

Unions of Lebesgue spaces and $A_{1}$ majorants

Greg Knese, John E. M ${ }^{\mathrm{C}}$ CARThy and Kabe Moen

Complex hyperbolic $(3,3, n)$ triangle groups

JOHN R. PARKER, JiEYAN WANG and BAOHUA XIE

Topological aspects of holomorphic mappings of hyperquadrics from $\mathbb{C}^{2}$ to $\mathbb{C}^{3}$

\section{MICHAEL REITER}

2-Blocks with minimal nonabelian defect groups III

BENJAMIN SAMBALE

Number of singularities of stable maps on surfaces 